\title{
Subset Optimization of Adaptive Coding and Modulation Schemes for Broadband Satellite Systems
}

\author{
V. Boussemart, H. Brandt and M. Berioli
}

\begin{abstract}
The number of Coding and Modulation Schemes suggested for the two standards DVB-S2 and DVB-RCS (in its "advanced" version) is clearly overdimensioned; a subset of schemes can be used by reducing the overall system performance negligibly. This paper presents the investigations performed on this rain fading mitigation technique, called Adaptive Coding and Modulation (ACM), supported by the DVB-S2 standard and shows how the sets of modulation and coding schemes, considered in the forward- and in the return-links, can be changed to reduce terminal complexity and how this affects the overall system performance. It is shown that, by a careful selection of the ACM schemes, already with less than one fourth (7 or 5 out of 28) of the total number of schemes it is possible to get performance close to the optimum, but with a much lower terminal complexity.
\end{abstract}

Index Terms-ACM, DVB-RCS, DVB-S2, ModCod, Simulator

\section{INTRODUCTION}

$\mathrm{B}$ ROADBAND satellite systems, based on geostationary (GEO) satellites in $\mathrm{Ku}$ and Ka bands, are particularly affected by atmospheric disturbances like the rain and clouds. Rain events modify the signal properties introducing scintillations, attenuations and depolarization [1]. These propagation effects directly influence the signal-to-noise ratio (SNR) creating fade events. The fade events produce errors in the transmission and techniques have to be developed to avoid them.

One fade mitigation technique (FMT), called adaptive coding and modulation (ACM), consists in modifying the coding and the modulation according to the current channel state. The combination of a modulation and a code (with a given code rate) is called mode or ModCod. Different ModCods can be used, going from the most robust one, i.e. the one offering poor spectral efficiency but robustness to fading, to the most efficient one, i.e. the one offering very high spectral efficiency but poor protection to fading

Manuscript received September 28, 2009. This work was realized using the satellite system simulator developed in the framework of the ESA funded project "Resources Management using Adaptive Fade Mitigation Techniques (FMT) in DVB-RCS Multi-Beam Systems" [6].

The authors are with the German Aerospace Center (DLR e.V.), Institute of Communications and Navigation, section Digital Networks, Oberpfaffenhofen, 82234 Wessling, Germany, phone: 0049 - (0)8153 28 2857, -2843, -2863; fax: 0049 - (0)8153 28 -2844; e-mail: \{vincent.boussemart; hartmut.brandt; matteo.berioli\}@dlr.de). transmission errors. The spectral efficiency is a measure expressed in bit/symbol/Hz influencing the long-term overall capacity available on the system. ACM was introduced in satellite systems to increase the system capacity [2]: efficient ModCods are used during clear sky conditions and robust ModCods during bad channel states (mainly due to rain events).

The Digital Video Broadcasting (DVB) Satellite Second Generation (DVB-S2 [3]) and Return-link Channel via Satellite (DVB-RCS [4]) standards define respectively the forward-link (from the gateway to the satellite terminals) and the return-link (from the satellite terminals to the gateway) ACM transmission schemes. The DVB-S2 standard supports ACM, DVB-RCS does not, for this reason a refinement of the DVB-RCS standard (called "advanced” DVB-RCS), including ACM, was recently defined by the European Space Agency ([5]). A powerful satellite system simulator, reproducing DVB-S2 and advanced DVB-RCS standards, was developed by the German Aerospace Center (DLR) and other partners in the framework of an ESA project [6] in order to make this type of studies and in particular to evaluate the impact of the ModCods considered on the overall system performance.

This paper presents the capabilities of the mentioned satellite system simulator in section II, then it describes the idea of the subset optimization of the ACM schemes in section III; the results and their analysis are presented in section IV, and the conclusions of the work are derived in section V.

\section{DVB-S2/RCS SIMULATOR DESCRIPTION}

The DVB-S2/RCS simulator consists of three main components: the pre-processing, mainly based on Matlab scripts, the simulator itself, which utilizes the object modular network testbed in $\mathrm{C}++(\mathrm{OMNeT}++[7])$, and the postprocessing, also mainly implemented in Matlab.

The satellite system considered in the simulator is based on a multi-beam (72 beams) GEO satellite with pan-European coverage. There are two different satellite configurations: the transparent (classical amplify and forward) one, and the regenerative one, with on-board processing and switching (packets arriving at the satellite are forwarded only to the beam in which the destination is located). The present analysis was performed on the transparent case, but results also applied to the regenerative case. 
Time series corresponding to the signal-to-noise and interference ratio (SNIR) over time at the satellite terminals are pre-computed with Matlab scripts; co-channel interference (CCI) is taken into account and modeled according to the location, the rain events and the traffic; a rain model [8] is used to generate the fading events for large-scale (thus wide area) as well as for short area (spatially correlated) rains. Scintillation (Fig. 1) and noise temperature increase (1)-(2) are added to obtain the resulting time series, as follows:

$T(t)=T_{C S}+T_{\Delta} \times\left(1-10^{-A(t) / 10}\right)$,

$N_{0}(t)=k \times T(t)$,

where $T_{C S}$ is the total clear sky temperature and $T_{\Delta}$ the maximum increase of the temperature due to rain.

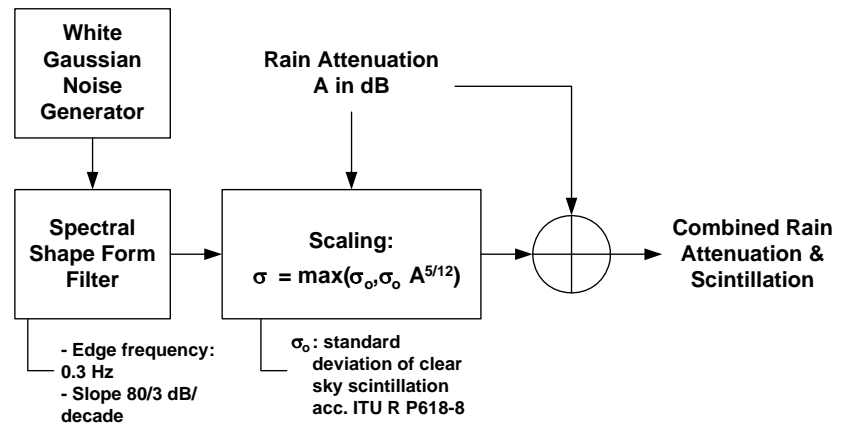

Fig. 1. Scintillation generation in the time series. Filtered white Gaussian noise (edge freq. $0.3 \mathrm{~Hz}(0.1-0.65 \mathrm{~Hz})$ ), 80/3 dB/decade slope, clear sky variance according to ITU P618-8. Scaled according to actual fade depth.

In order to use ACM channel measurements have to be performed in reality and this is simulated also. The channel measurements are realized by the satellite terminals while receiving the forward-link signal and by the gateways while receiving the return-link signal. Due to the inherent delay (greater than $500 \mathrm{~ms}$ ) between the channel estimation and the application of a new ModCod, "safety margins" are introduced. Actually ACM operates as follows: if the SNIR is above a given threshold then a more efficient ModCod can be selected, if it is below then a more robust ModCod has to be considered. A hysteresis is introduced in the mode selection so that an oscillation between two modes does not occur [9]. Different thresholds are created for a given ModCod mainly to switch to higher or lower modes.

The forward-link is organized following the time-division multiplexing scheme (TDM). Data and signaling are selected and encapsulated by the gateway scheduler using an algorithm respecting the QoS, the service level agreement (SLA) and the ModCod attribution ("pool scheduling" algorithm [10]). Baseband frames (BBFRAMES) are produced, are encoded into FECFRAMES and are modulated into physical-layer frames (PLFRAMES) before being transmitted to the satellite.

The allocation of the resources on the return-link is performed using the multi-frequency - time division multiple access (MF-TDMA). Slots are attributed to users by the return-link resource manager (RRM) located into the gateway. The resources are organized in both frequency and time slots in the terminal-burst time plan (TBTP) and are assigned to satellite terminals by the gateway.

\section{SubSet Optimization OF AdAPtive Coding AND MODULATION SCHEMES}

The way the time series are produced, the thresholds are computed and the modes are selected is of paramount importance when using adaptive coding and modulation (ACM). In the previous section the generation of the time series was described, detailing how the scintillation and noise temperature increase were taken into account. Actually the scintillation only involves very rapidly varying distortions, which are superimposed to slowly varying distortions (due to rain events), for this reason scintillation impacts directly the value of the safety margins introduced in the ModCod thresholds.

Two thresholds are mandatory in the forward-link: the upthreshold and the down-threshold. For each ModCod but the last one the up-threshold indicates the SNIR value above which the mode needs to be upgraded. For each ModCod but the first one the down-threshold defines the SNIR value below which the current ModCod requires to be downgraded. For the purpose of this work, in the forward-link 19 modes out of the 28 ModCods defined by the DVB-S2 standard are used (see Table I). Some ModCods were not considered since they overlap with other ones. The SNIR critical thresholds shown in Table I are the SNIR values required to achieve in each ModCod a worst-case PER equal to $10^{-7}$. The SNIR subscript indicates what is the packet type (MPEG-TS, FBL400, or FBL800) considered to calculate the threshold.

TABLE I. FL (LEFT) AND RL (RIGHT) MODCODS CONSIDERED.

\begin{tabular}{|c|c|c|c|}
\hline ID & Mod. & $\mathrm{R}$ & SNIR $_{\text {MPEG-TS }}$ \\
\hline 1 & QPSK & $1 / 4$ & $-0,25$ \\
\hline 2 & QPSK & $1 / 3$ & 0,86 \\
\hline 3 & QPSK & $2 / 5$ & 1,8 \\
\hline 4 & QPSK & $1 / 2$ & 3,1 \\
\hline 5 & QPSK & $3 / 5$ & 4,33 \\
\hline 6 & QPSK & $2 / 3$ & 5,2 \\
\hline 7 & QPSK & $3 / 4$ & 6,13 \\
\hline 8 & QPSK & $4 / 5$ & 6,78 \\
\hline 9 & QPSK & $5 / 6$ & 7,28 \\
\hline & & & \\
\hline 10 & $8 P S K$ & $3 / 5$ & 8,25 \\
\hline 11 & $8 P S K$ & $2 / 3$ & 9,37 \\
\hline 12 & $8 P S K$ & $3 / 4$ & 10,66 \\
\hline & & & \\
\hline 13 & $8 P S K$ & $5 / 6$ & 12,1 \\
\hline 14 & 16 APSK & $2 / 3$ & 13,8 \\
\hline 15 & 16 APSK & $3 / 4$ & 15 \\
\hline 16 & 16 APSK & $4 / 5$ & 15,8 \\
\hline 17 & 16 APSK & $5 / 6$ & 16,4 \\
\hline & & & \\
\hline 18 & $16 A P S K$ & $8 / 9$ & 17,7 \\
\hline 19 & 16 APSK & $9 / 10$ & 18 \\
\hline
\end{tabular}

\begin{tabular}{|c|c|c|c|c|}
\hline $\mathrm{ID}$ & Mod. & $\mathrm{R}$ & SNIR $_{\text {FBL400 }}$ & SNIR $R_{\text {FBL800 }}$ \\
\hline & & & & \\
\hline 1 & QPSK & $1 / 3$ & 1,73 & 1,02 \\
\hline & & & & \\
\hline 2 & QPSK & $1 / 2$ & 3,84 & 3,17 \\
\hline 3 & QPSK & $3 / 5$ & 4,94 & 4,36 \\
\hline 4 & QPSK & $2 / 3$ & 5,76 & 5,17 \\
\hline 5 & QPSK & $3 / 4$ & 6,8 & 6,22 \\
\hline 6 & QPSK & $4 / 5$ & 7,51 & 6,92 \\
\hline 7 & QPSK & $5 / 6$ & 8,04 & 7,44 \\
\hline 8 & QPSK & $6 / 7$ & 8,45 & 7,85 \\
\hline & & & & \\
\hline 9 & $8 P S K$ & $2 / 3$ & 8,84 & 8,4 \\
\hline 10 & 8 PSK & $3 / 4$ & 10,11 & 9,67 \\
\hline 11 & 8 PSK & $4 / 5$ & 11,05 & 10,63 \\
\hline 12 & 8 PSK & $5 / 6$ & 11,53 & 11,11 \\
\hline & & & & \\
\hline 13 & 16 APSK & $3 / 4$ & 12,02 & 11,68 \\
\hline 14 & 16 APSK & $4 / 5$ & 12,97 & 12,68 \\
\hline 15 & 16 APSK & $5 / 6$ & 13,45 & 13,17 \\
\hline 16 & 16 APSK & $6 / 7$ & 13,92 & 13,84 \\
\hline 17 & 16 APSK & $8 / 9$ & 14,66 & 14,42 \\
\hline 18 & 16 APSK & $9 / 10$ & 14,81 & 14,58 \\
\hline
\end{tabular}

For the return-link the situation is a bit more complex. The gateway receives the bursts transmitted by the satellite terminals. It measures the current channel state for a given terminal and uses several criteria to verify whether the mode selected requires to be modified. Because of the bursty structure of the transmission (measurements can be made only if the satellite terminal transmits data), there are three ModCod threshold types in the return-link. Both up- and down-thresholds are still present but a new threshold named 
"decision threshold" is necessary. Actually the values of the three thresholds are not only a function of the ModCod but also of the time and of the burst type. The time is relevant since the ModCod selection depends on the age of the last measurement received. The values of the thresholds also depend on the burst type (fixed information length or fixed burst length) and on the burst size. The decision thresholds are used for initial ModCod selection (first measurement received) or when the last measurement is too old. Eighteen ModCods are utilized in the return-link (Table I).

ModCod subsets are specific selections of groups of modes, i.e. a choice of ModCods extracted from the complete list (Table I). The ModCod subsets are built based on the approach given in [11] and by considering the values of the SNIR critical thresholds.

In the forward-link the values of the SNIR thresholds are obtained for $\mathrm{PER}<10^{-7}$ and a packet length equal to 188 bytes (MPEG-TS packet). SNIR thresholds for the forward-link are given in [3], but they do not take into account the implementation losses related to the modulation scheme and the total losses due to the satellite non-linearity, so the ones presented in Table I were re-computed taking into account these new factors. The non-linearity is caused by the output back-off and depends on the modulation scheme and on the number of used carriers (in the simulations two carriers were considered for the forward-link).

In the return-link a fixed burst length (FBL) configuration is considered with burst length 400 symbols and 800 symbols (FBL400 and FBL800 in Table I). In such a case the number of symbols is fixed and the number of bits that can be inserted depends on the ModCod scheme. The required SNIR is computed taking into account guard symbols and preamble/pilot symbols. The number of guard symbols is fixed and equal to four: two guard symbols at the beginning and two guard symbols at the end of the burst. The number of preamble/pilot symbols depends on the burst length. When considering FBL400 the preamble/pilot (pp) sequence represents $p_{\mathrm{pp}}=15 \%$ of the physical layer (PHY) payload (without the PHY guard symbols). When considering FBL800 the preamble/pilot sequence represents $p_{\mathrm{pp}}=10 \%$ of the PHY payload without guard symbols. The net number of bits available for inserting the payload is thus computed using the next formula:

$b_{\text {payload }}=\frac{S_{\mathrm{PHY}}-S_{\mathrm{PHY}-\mathrm{G}}}{1+p_{\mathrm{pp}}} \times M \times R$,

where $S_{\mathrm{PHY}}$ represents the total number of symbols in the PHY layer burst, $S_{\text {PHY G }}$ the PHY guard symbols, $M$ the number of bits per symbol, $R$ the code rate, and $p_{\mathrm{pp}}$ the share of preamble/pilot symbols with respect to the PHY payload without guard symbols. The SNIR thresholds for the returnlink were obtained by interpolating values according to [12] and [13] and are summarized in Table I.

The ModCod subsets are created in the following way. First SNIR thresholds are expressed as functions of the ModCod
IDs: $\operatorname{SNIR}_{\mathrm{FL}}$ (ID) for the forward-link and $\mathrm{SNIR}_{\mathrm{RL}}$ (ID) for the return-link. Then two linear approximations of these functions are derived using the least mean square method: $\mathrm{SNIR}_{\mathrm{FL}, \text { lin }}(\mathrm{ID})$ and $\mathrm{SNIR}_{\mathrm{RL}, \mathrm{in}}(\mathrm{ID})$. These functions represent approximations to the real values of the SNIR thresholds for each ModCod ID. If $m$ is the number of ModCods that should be in the subset, then the SNIR distance between the two extreme ModCods is divided into $m-1$ portions and $m$ SNIR* values at the edges of the $m-1$ portions are derived. Then the linear functions $\mathrm{SNIR}_{\mathrm{FL}, \text { lin }}(\mathrm{ID})$ and $\mathrm{SNIR}_{\mathrm{RL}, \text { lin }}(\mathrm{ID})$ are inverted to derive (from the $m$ SNIR* values) the ModCod IDs that are the closest to these SNIR* values. Fig. 2 shows this ModCod subset selection where the number of desired ModCod is seven.

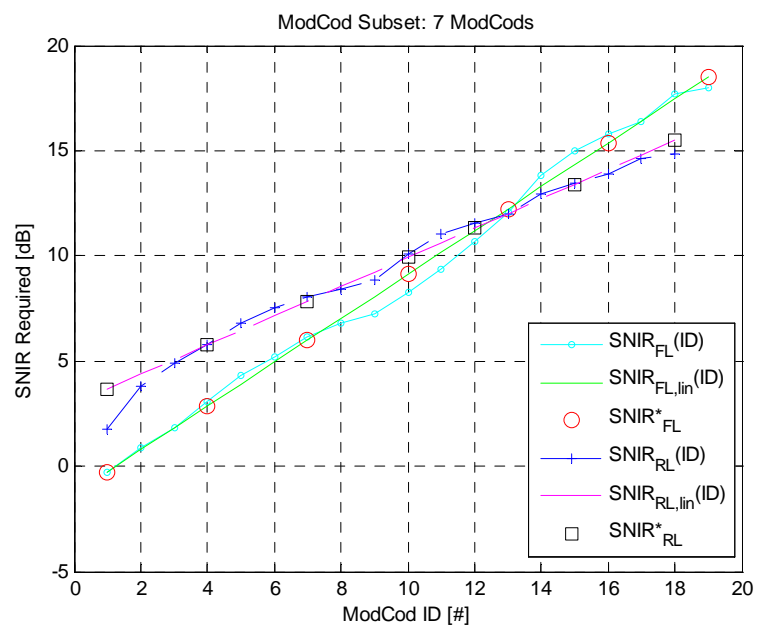

Fig. 2. FL and RL ModCod subset selection result while targeting 7 modes.

TABLE II. FL (LEFT COLUMNS) AND RL (RIGHT COLUMNS) MODCOD SUBSETS.

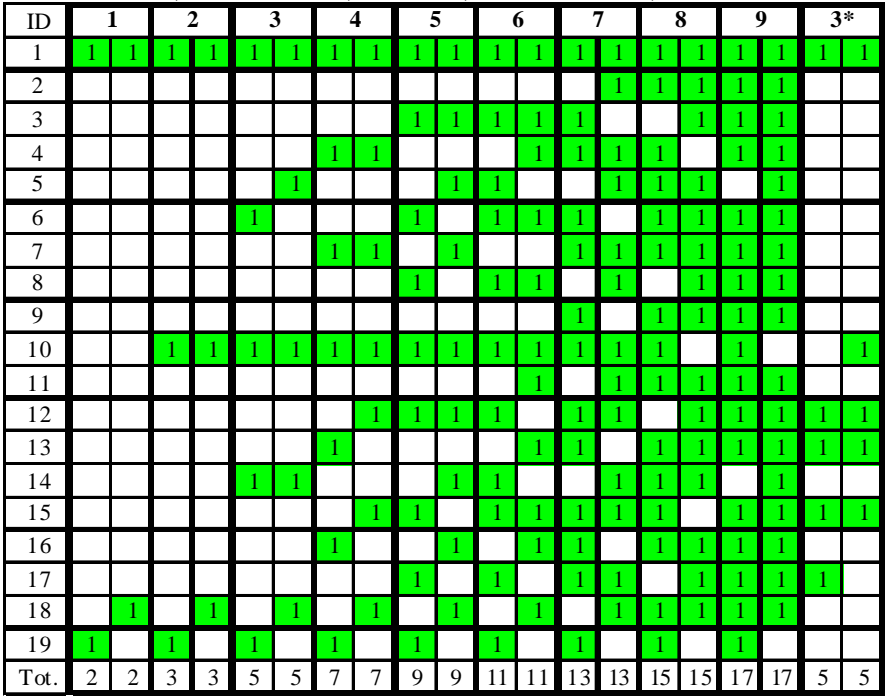

The subset 1 is simply built by taking into account the most robust ModCod and the most effective one. The following eight subsets (2 to 9) are only composed by an odd number of ModCods for both FL and RL and were obtained using the method described previously. The last subset $\left(3^{*}\right)$ is an extra subset in which ModCods were selected according to other empiric optimization criteria explained in the next section. The columns in Table II represent the 10 ModCod subsets. 
"1" indicates that the ModCod ID (rows in the table) is considered. The ModCod subset composition is given for each ModCod subset for the forward-link (left columns) and the return-link (right columns). As it can be seen in Table II the ModCod subsets were built so that the number of ModCods represented by a particular subset ID is the same for the forward-link and the return-link.

\section{Simulation Results}

This part of the document presents the results of the computer simulations performed with the ModCod subsets. The system was overloaded with a high number of terminals in order to properly evaluate the performance of the algorithms.

The resulting ModCod distribution in both forward- and return-links is given by Fig. 3. It can be observed that the most robust mode in the FL is used to transport signaling, but for most of the time QPSK is not selected since the channel state is good enough to use more efficient modes. Less efficient ModCods are selected in the RL compared to the FL since the link budgets are different.

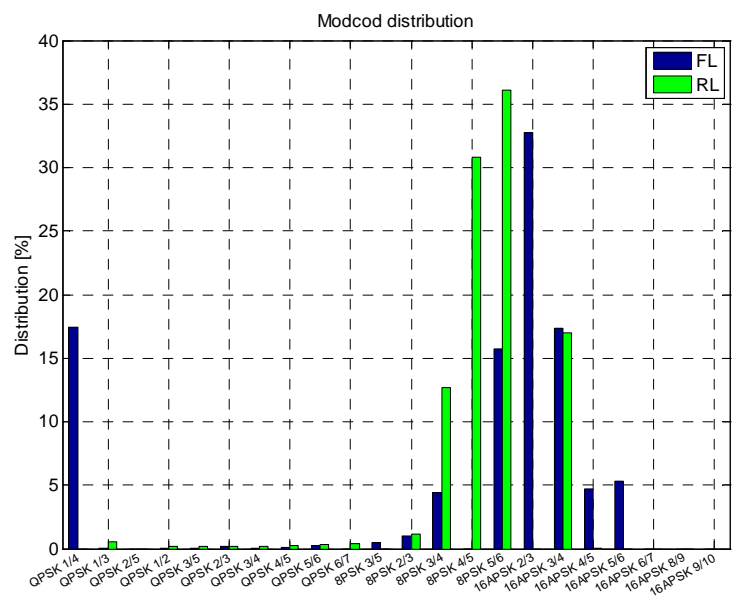

Fig. 3. FL and RL ModCod distribution. The 22 ModCods are represented (Table I). In the FL the distribution is computed among the short and long frames transmitted. In the RL it corresponds to the distribution of the updates (mode change) sent by the terminal decoder (gateway).

As it is well known in literature, the use of ACM can guarantee impressive gains in both links, and this is confirmed by simulations ([14]): about $50 \%$ of capacity increase in the FL and about $100 \%$ in the RL. Also the use of novel encapsulation schemes (GSE instead of MPE) involves a better physical efficiency (gain of about $10 \%$ for both FL and RL mainly due to less overhead).

Simulations were performed to test the different ModCod subsets using ACM in FL and in RL (Table II). For each ModCod subset a configuration was created and a simulation was run. Among all measurements available in the simulator the IP efficiency was selected as the most relevant. In the FL it corresponds to the number of bits in IP packets (including headers) transmitted by the gateway, divided by the number of symbols within a useful PHY frame. In the RL it is computed as the number of bits in IP packets (including headers) transmitted by the satellite terminal divided by the number of PHY symbols in the slots used by the terminal. The IP efficiency is thus expressed in bits/symbol and, if the system is overloaded, it can be used as a fair measure of the system capacity across different simulations; thus the higher the IP efficiency, the higher the capacity of the system.

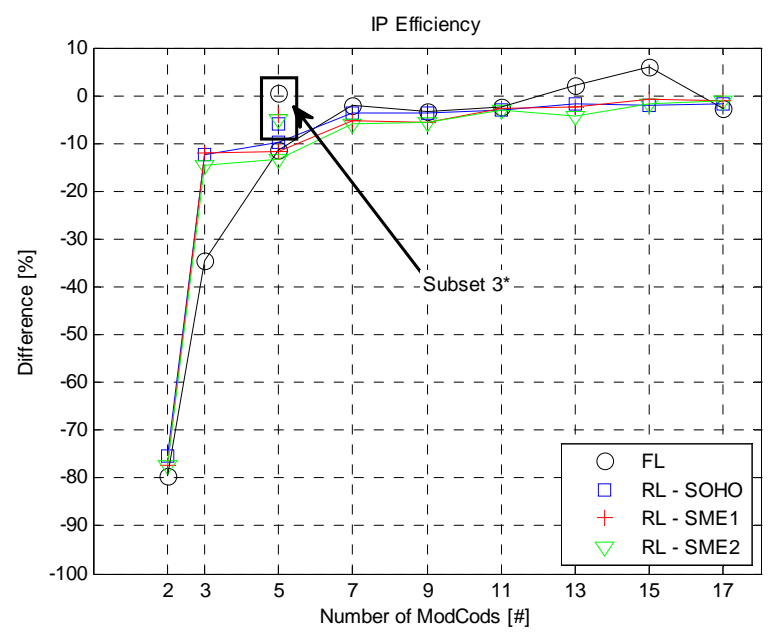

Fig. 4. FL and RL IP efficiency for the different ModCod subsets. The mean value of the IP efficiency is computed over the time during which the system is stable (i.e. after one simulated-hour). The difference is calculated with respect to the mean value obtained when all ModCods are considered.

In the FL the utilization of only two modes provides very bad results (Fig. 4) simply because the most efficient ModCod is never used. Using three ModCods shows better results since an intermediate mode (8PSK 3/5) can be selected. The principle remains the same with five modes where ModCod 16APSK 2/3 can be used (see Fig. 3). The next subset (no. 4) presents an IP efficiency almost identical to the reference: seven ModCods are considered instead of nineteen. Using nine and eleven ModCods shows a (small) degradation in terms of performances unlike using thirteen and fifteen. Such a difference can be explained by the utilization of ModCod ID 16 (16APSK 4/5). The last subset (seventeen modes) shows a small decrease of the efficiency (about 3\%) even if almost all ModCods are considered. The way the FL IP efficiency evolves according to the ModCod subset is related to several factors. First the distribution of the ModCod is of paramount importance when all modes are taken into account (Fig. 3). The distribution is influenced by the link budget between the gateway and the satellite terminals and by the weather conditions. Second the efficiency depends also on the scheduling algorithm. The scheduling algorithm adopted has knowledge of the ModCods attributed on the FL and tries to optimize the construction of the BBFRAMES according to that. When the number of ModCods gets lower the probability of having identical ModCods between satellite terminals is higher. This favors the construction of BBFRAMES which are better filled and directly involves an increase of the capacity. The last subset (subset $3^{*}$ ) is a refinement of subset 3 (five modes) and was built so that implementation complexity and costs are reduced. This subset considers only three modulation schemes (QPSK, 8PSK, and 16APSK) and three code rates 
(1/4, 3/4, and 5/6). QPSK 1/4 (mandatory for signaling) will be selected in bad channel conditions, 8PSK 3/4 and 5/6 in transient states and 16APSK $3 / 4$ and 5/6 in good weather conditions. Subset $3 *$ thus consists of five ModCods and the IP efficiency is almost identical to the one obtained with 19 ModCods (difference less than $1 \%$ ).

The results for the return-link depend on the terminal type, due to the different link budgets (see Fig. 4). As in the FL, the first subset (with two modes) presents bad IP efficiency: the most efficient ModCod (mode 18) is never attributed (Fig. 3). The efficiency already increases dramatically while considering three ModCods. The IP efficiency then goes higher with the other ModCod subsets and starting from using seven modes the difference between satellite terminal types with respect to the scenario with all ModCods is almost identical (around 5\%). As mentioned before the difference in efficiency between the satellite terminal types can be explained by their link budgets. SOHO terminals use smaller antennas than SME1, which on the other hand use smaller antennas than SME2. The antenna size directly impacts the link budget and thus the signal-to-noise ratio. This results into different ModCod distributions among the terminals. The ModCod subsets thus have different effects on the terminals and this is shown by Fig. 4. Moreover all ModCods having IDs above 14 are almost never attributed (Fig. 3) and this explains the quasi stability of the IP efficiency starting from subsets with more than seven modes. The scheduling algorithm does not play a major role on the IP efficiency in the RL since only one ModCod is considered for a given terminal, whereas in the FL the gateway has to take into account several ModCods since it communicates with terminals experiencing various channel conditions. The last subset (subset $3^{*}$ ) is based on the same principle than the last subset in the FL: it represents a refinement of subset 3 and a compromise between efficiency and complexity. This subset also presents only three modulation schemes (QPSK, 8PSK, and 16APSK) and three code rates (1/3, 3/4, and 5/6), used to build five ModCods. QPSK 1/3, the most robust one, can be selected in case of bad channel conditions, 8PSK and 16APSK modulations for better channel condition. The IP efficiency obtained with these five modes is almost identical to the one obtained with greater number of modes (difference of about $5 \%$ ).

\section{CONCLUSION}

This paper showed the possibility of using optimized sets of ACM schemes in recently standardized broadband satellite systems, both on the forward-link (DVB-S2) and the returnlink ("advanced" DVB-RCS). These standards presents today a wide range of ACM schemes (also named ModCods), but it is meaningful, in order to reduce the complexity and the costs of the satellite terminals, to constraint the number of implemented ModCod. The results have shown that with less than one fourth of the total number of ModCods ( 5 out of the 28 ones defined in the DVB-S2 standard [3]), one can reach almost the same overall system capacity as with the full set of ModCods. The aim of optimizing subsets of ACM schemes is of course to produce cheaper satellite terminals (with less sophisticated hardware), but also to simplify the resource management in the overall system, since the problem of selecting the best ModCod becomes much simpler when the number of ModCods available is low.

\section{ACKNOWLEDGEMENT}

The authors thank the consortium of the ESA Study 18826/05/NL/US in which they took part and which consisted of TriaGnoSys GmbH (project leader), Audens ACT, German Aerospace Center (DLR) and Nera. The work presented in this paper is based on the simulator developed for this ESA Study. The authors modified the simulator and implemented additional parts in order to make these investigations on ACM.

\section{REFERENCES}

[1] G. Maral, M. Bousquet. "Satellite Communications Systems”, Fourth Edition, 2002, pp. 223-241.

[2] R. Rinaldo and R. De Gaudenzi, "Capacity analysis and system optimization for the forward link of multi-beam satellite broadband systems exploiting adaptive coding and modulation”, International Journal of Satellite Communications and Networking, vol. 22, pp. 401423, issue 3, June 2004.

[3] Digital Video Broadcasting (DVB): "Second generation framing structure, channel coding and modulation systems for broadcasting, interactive services, new gathering and other broadband satellite applications”, European Standard (Telecommunications series), ETSI EN 302307 V1.1.2, 2006-06.

[4] Digital Video Broadcasting (DVB): "Interaction channel for satellite distribution systems", European Standard (Telecommunication series), ETSI EN 301790 V1.5.1, 2009-01.

[5] ESA funded project "Protocols and Signalling for Adaptive Fade Mitigation Techniques (FMT) in DVB-RCS Multi-Beam Systems”. ESA Study 17403/03/NL/ND, 2003,

http://telecom.esa.int/telecom/www/object/index.cfm?fobjectid=22703.

[6] ESA funded project "Resources Management using Adaptive Fade Mitigation Techniques (FMT) in DVB-RCS Multi-Beam Systems”. ESA Study 18826/05/NL/US, 2005,

http://telecom.esa.int/telecom/www/object/index.cfm?fobjectid=17247.

[7] A. Varga, "The OMNET++ Discrete Event Simulation System", Proceedings of the European Simulation Multiconference (ESM'2001), Prague, Czech Republic, June 6-9, 2001.

[8] B. Grémont, "Simulation of Rainfield Attenuation for Satellite Communication Networks”, Cost 280, PM3014, Jul. 2002.

[9] T. Wörz, R. Schweikert, A. Jahn amd R. Rinaldo, "Physical layer efficiency of satellite DVB using fade mitigation techniques”, Proceedings of the International Communication Satellite Systems Conference (ICSSC'05), Rome, Italy, Sept. 2005.

[10] C. Párraga Niebla, "Scheduling Techniques for Satellite Systems with Adaptive Coding and Modulation”, Proceedings of ASMS Conference, Herrsching, Germany, May 29-31, 2006.

[11] M. Smolnikar, T. Javornik, M. Mohorcic and M. Berioli, "DVB-S2 Adaptive Coding and Modulation for HAP Communication System”, Vehicular Technology Conference (VTC), May 11-14, 2008.

[12] Digital Video Broadcasting (DVB): "Interaction channel for Satellite Distribution Systems; Guidelines for the use of EN 301 790”, ETSI TR 101790 V1.1.1, 2001-09.

[13] http://www-elec.enst-bretagne.fr/turbo/LIMIT/ (activate Java and Java Script).

[14] V. Boussemart and H. Brandt, "Performance Assessment using ACM in DVB-S2/Advanced DVB-RCS Satellite Systems", Proceedings of the International Communication Satellite Systems Conference (ICSSC'09), Edinburgh, UK, June. 2009. 